\title{
The Cryptanalysis of Reduced-Round SMS4
}

\author{
Jonathan Etrog* and Matt J.B. Robshaw \\ Orange Labs \\ 38-40 rue du Général Leclerc \\ 92794 Issy les Moulineaux Cedex 9, France \\ \{forename.surname\}@orange-ftgroup.com
}

\begin{abstract}
In this paper we consider the cryptanalysis of the block cipher SMS4. The cipher has received much recent attention due its simplicity and prominence (it is used in wireless networks in China) and a range of differential attacks break up to 21 of the 32 rounds used in SMS4. Here we consider the application of linear cryptanalysis to the cipher and we demonstrate a simple attack on 22 rounds of SMS4. We also consider some advanced linear cryptanalytic techniques which, under the best conditions for the cryptanalyst, might (just) extend to 23 rounds.
\end{abstract}

\section{Introduction}

In this paper we consider the security of the block cipher SMS4 which is reputedly mandated for wireless networks in China [10. A Chinese description of the cipher was made public in 2006 by the Chinese government, and the first analysis in the open community was published in 2007 [10]. The cipher takes a 128-bit block and key, and it consists of 32 simple rounds. Its intriguing design encourages analysis; something which is due in no small part to the fact that minor variants of the cipher are exceptionally weak.

The first open analysis of a reduced-round version of SMS4 examined the algebraic nature of the algorithm - thereby uncovering the construction of the S-box - and yielded a saturation attack over 13 rounds using $2^{16}$ chosen plaintext pairs and $2^{114}$ operations [10]. This was followed by a differential attack on 14 rounds and then by an impossible differential attack on 16 rounds with the claimed requirements of $2^{105}$ chosen plaintext pairs and $2^{107}$ operations [11]. These are rather complex attacks, and a more natural differential attack has been revealed that suggests that 21 rounds could be compromised using $2^{118}$ chosen plaintext pairs and $2^{126.6}$ operations $[22$. This is the previous best known attack in the literature.

In this paper we present the first reported application of linear cryptanalysis to SMS4. Apart from DES [15, there are few ciphers for which linear cryptanalysis yields a more efficient attack than differential cryptanalysis. However, for SMS4 we propose an attack on 22 rounds of the cipher with less than $2^{119}$ known

^ Partially supported by the national research project RFIDAP ANR-08-SESU-009-03.

R. Avanzi, L. Keliher, and F. Sica (Eds.): SAC 2008, LNCS 5381, pp. 51 65, 2009.
(C) Springer-Verlag Berlin Heidelberg 2009 
plaintexts and a work effort roughly equivalent to $2^{117}$ 22-round SMS encryptions. The attack can be clearly described and the necessary components have been experimentally verified. We also consider attacks on 23 rounds of SMS4 and highlight some future research directions.

\section{Description of SMS4}

We briefly describe the block cipher SMS4, but first we establish our notation.

Notation. For the most part we will be working with 32-bit words, though the context will be clear when we restrict ourselves to bytes. The left rotation (resp. right rotation) of a word $x$ by $b$ bit positions will be denoted $x \lll b($ resp. $x \ggg b)$. The remaining notation is standard in the cryptographic literature.

Encryption and Decryption. SMS4 is a 32-round block cipher with a 128-bit key and block. It is an unbalanced Feistel cipher, that repeatedly uses an 8-bit Sbox $S$. This is described in the appendices and it is, by way of construction [10], closely related to the AES S-box [16]. We define the $L$ function and the $\gamma$ function as follows

$$
\begin{aligned}
L(x) & =x \oplus(x \lll 2) \oplus(x \lll 10) \oplus(x \lll 18) \oplus(x \lll 24) \\
\gamma(x) & =\left(S\left[x_{31 \ldots 24}\right]\left\|S\left[x_{23 \ldots 15}\right]\right\| S\left[x_{15 \ldots 8}\right] \| S\left[x_{7} \ldots 0\right]\right) .
\end{aligned}
$$

The action of the round function $f$ on input $X_{i-1}$ to the $i^{\text {th }}$ round of SMS4 is given by $f\left(X_{i-1}\right)=L\left(\gamma\left(X_{i-1} \oplus k_{i}\right)\right.$. Two rounds of SMS4 are shown in Figure 1

\begin{tabular}{|c|c|c|c|c|c|c|c|c|c|c|c|c|c|c|c|c|}
\hline & & -1 & -2 & -3 & -4 & 5 & -6 & -7 & -8 & -9 & $-a$ & $-b$ & $-c$ & $-d$ & $-e$ & $-f$ \\
\hline D- & $\mathrm{d} 6$ & 90 & $\overline{\mathrm{e} 9}$ & $\mathrm{fe}$ & $C \mathrm{CC}$ & 21 & $3 d$ & b7 & 16 & b6 & 14 & $\mathrm{c} 2$ & 28 & $\mathrm{fb}$ & $2 c$ & $\sqrt{5}$ \\
\hline $1-$ & $2 b$ & 67 & $9 a$ & 76 & $2 a$ & be & 04 & $c 3$ & aa & 44 & 13 & 26 & 49 & 86 & 06 & 95 \\
\hline$z-$ & 90 & 42 & 50 & 4 & 91 & ef & 98 & $\mathrm{a}$ & 33 & 54 & Ob & 43 & ed & CI & $\mathrm{ac}$ & 62 \\
\hline $3-$ & e4 & b3 & 1c & a9 & 9 & 08 & e8 & 5 & 80 & $\mathrm{df}$ & 94 & 土a & 10 & $3 f$ & 31 & $\mathrm{ab}$ \\
\hline $4-$ & 47 & 07 & a7 & $\mathrm{fC}$ & f3 & 73 & 17 & ba & 83 & 59 & $3 c$ & 19 & e6 & 5 & $4 \mathrm{f}$ & $a \varepsilon$ \\
\hline $5-$ & 68 & $6 \mathrm{~b}$ & 81 & b2 & 1 & 64 & 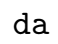 & b & f8 & eb & of & $4 \mathrm{~b}$ & 70 & 6 & $9 d$ & 35 \\
\hline $6-$ & $1 \mathrm{e}$ & 24 & $\mathrm{Oe}$ & $5 e$ & 3 & 58 & d1 & 2 & 25 & 22 & $7 \mathrm{c}$ & $3 b$ & 01 & 21 & $7 \varepsilon$ & 87 \\
\hline 7- & d4 & 00 & 46 & 7 & $y$ & d3 & 27 & 2 & $4 c$ & 36 & 02 & e7 & $\mathrm{a} 0$ & c4 & C8 & $9 e$ \\
\hline 8- & ea & $\mathrm{bf}$ & $8 a$ & $\mathrm{~d} 2$ & 40 & c7 & 38 & 5 & a3 & f7 & f2 & $\mathrm{ce}$ & f9 & 61 & 1 & a. \\
\hline 9- & $\mathrm{e} 0$ & ae & $5 d$ & a4 & $9 b$ & 34 & $1 \mathrm{a}$ & 5 & $\mathrm{ad}$ & 93 & 32 & 30 & f5 & $8 c$ & $\mathrm{~b} 1$ & e3 \\
\hline$a-$ & $1 d$ & f6 & e2 & $2 e$ & 82 & 66 & $\mathrm{ca}$ & 0 & c0 & 29 & 23 & $a b$ & $0 d$ & 53 & $4 e$ & $6 \mathrm{f}$ \\
\hline D- & d5 & $\mathrm{db}$ & 37 & 45 & $\mathrm{de}$ & Is & $8 \in$ & $2 f$ & J3 & \pm \pm & $6 a$ & 12 & $6 \mathrm{~d}$ & bc & $b b$ & 51 \\
\hline E- & 80 & It & $\mathrm{af}$ & 92 & & $\mathrm{dd}$ & $\mathrm{bc}$ & $7 f$ & 11 & d9 & $5 c$ & 41 & $1 \mathrm{f}$ & 10 & ba & $\mathrm{d} \varepsilon$ \\
\hline$x-$ & $0 \mathrm{a}$ & c1 & 31. & 88 & & $\mathrm{~cd}$ & $7 \mathrm{~b}$ & $\mathrm{bd}$ & $2 \mathrm{~d}$ & 74 & do & 12 & b8 & e5 & b4 & bo \\
\hline & 89 & 68 & 97 & $4 \pi$ & U & 96 & 11 & $7 e$ & 65 & b9 & f1 & 09 & $c b$ & $6 e$ & c6 & 84 \\
\hline & & 1 & & & & & & & & & $5 I$ & & at & $C D$ & $3=$ & $4 \varepsilon$ \\
\hline
\end{tabular}

\section{The SMS4 S-box}


The Key Schedule. The key schedule is similar to the encryption function. Each subkey $k_{i}$ is derived as one word from the output of a single round of SMS-like encryption where the "key" for each round $i$ is a constant $g(i)$ (to be defined below). The plaintext for the start of the key generation is the 128-bit user-supplied key $K_{[127 \ldots 0]}$. The round function for the SMS-like encryption is given by

$$
\begin{aligned}
L^{\prime}(x) & =x \oplus(x \lll 13) \oplus(x \lll 23) \\
\gamma(x) & =\left(S\left[x_{31 \ldots 24}\right]\left\|S\left[x_{23 \ldots 15}\right]\right\| S\left[x_{15 \ldots 8}\right] \| S\left[x_{7 \ldots 0}\right]\right)
\end{aligned}
$$

so only the L-function is changed in comparison with encryption. At the start, the user-supplied key is xor-ed with a constant

$$
T=0 x a 3 b 1 \text { bac6 0x56aa3350 0x677d9197 0xb27022dc, }
$$

and the initialization of the generation of the subkeys 11 is as follows:

$$
\begin{array}{cl}
k_{-3}=K_{[127 \ldots 96]} \oplus T_{[127 \ldots 96]}, & k_{-2}=K_{[95 \ldots 64]} \oplus T_{[95 \ldots 64]}, \\
k_{-1}=K_{[63 \ldots 32]} \oplus T_{[63 \ldots 32]}, & k_{0}=K_{[31 \ldots 0]} \oplus T_{[31 \ldots 0]} .
\end{array}
$$

The key $k_{i}$ for the $i^{\text {th }}$ round, for $1 \leq i \leq 32$ is computed as

$$
k_{i}=k_{i-4} \oplus L^{\prime}\left(\gamma\left(k_{i-3} \oplus k_{i-2} \oplus k_{i-1} \oplus g(i)\right)\right)
$$

where each constant $g(i)$ is defined by

$g(i)=((28 \times(i-1))\|(28 \times(i-1)+7)\|(28 \times(i-1)+14) \|(28 \times(i-1)+21))$.

\subsection{RED-SMS4: A Small Version of SMS4}

We confirm some of the work in this paper with experiments, and for these we will need to define a reduced-version of SMS4. This will be a block cipher with a 64-bit key and block size which uses a 4 -bit S-box $S_{r}$. For experiments we chose the S-box used in PRESENT [2]. We can define a reduced $L_{r}$ function and a reduced $\gamma_{r}$ function as follows:

$$
\begin{aligned}
L_{r}(x) & =x \oplus(x \lll 8) \oplus(x \lll 10) \\
\gamma_{r}(x) & =\left(S_{r}\left[x_{15 \ldots 12}\right]\left\|S_{r}\left[x_{11 \ldots 8}\right]\right\| S_{r}\left[x_{7 \ldots 4}\right] \| S_{r}\left[x_{3 \ldots 0}\right]\right) .
\end{aligned}
$$

$L_{r}$ was built using the rotations that appear in $L$ modulo 16 . In this way, the round function $f_{r}$ used in the $i^{\text {th }}$ round of reduced-SMS4 is given by $f_{r}\left(X_{i-1}\right)=$ $L_{r}\left(\gamma_{r}\left(X_{i-1} \oplus k_{i}\right)\right.$. A reduced version of the key schedule requires us to change the linear function $L^{\prime}$ to $L_{r}^{\prime}$ just as we changed $L$ to $L_{r}$ in the encryption routine, and to revise the per-round constants to $g_{r}(i)=((28 \times(i-1)) \|(28 \times(i-1)+7))$.

${ }^{1}$ This is slightly different to other descriptions so as to accommodate the natural numbering of rounds starting with 1. 


\section{Linear Cryptanalysis}

While linear cryptanalytic methods appeared in [21, the linear cryptanalytic attack and its application to DES was developed by Matsui [1213. The basic idea is to find a linear approximation to the action of the block cipher. By this we mean a linear equation that includes $a$ bits of the plaintext $P_{r_{1}}, \ldots, P_{r_{a}}$, together with $b$ bits of the ciphertext $C_{s_{1}}, \ldots, C_{s_{b}}$ and a single bit of key-related information $\kappa$. Borrowing the vector inner-product, we will use the notation $\alpha \cdot P$ to denote the sum of plaintext bits $P_{r_{1}} \oplus \ldots \oplus P_{r_{a}}$ where $\alpha=\sum_{j=1}^{a} 2^{r_{j}}$ and $\alpha$ is called a linear mask. We will then write a single linear approximation as

$$
\alpha \cdot P \oplus \beta \cdot C=\kappa \text {. }
$$

If $\kappa$ (the exclusive-or of subkey bits) is fixed, then Equation 1 will be correct with probability $p=\frac{1}{2}+\epsilon$ and we say that the linear approximation has a bias of $\epsilon$. Given a bias of sufficiently large absolute value $|\epsilon|$ and sufficiently many known plaintext/ciphertext pairs, the value of $\kappa$ can be deduced thereby revealing one bit of key information. Throughout the paper the term "bias" will refer to its absolute value.

It is well-known that we can recover more bits of the key by using Matsui's Algorithm 2 [12. Here we use a linear approximation over several inner rounds, say rounds $b$ to $c$ of the $r$-round cipher, and this approximates one inner bit of key information (which is a function of the subkeys $k_{b}, \ldots, k_{c}$ ). Since the inputs to this linear approximation are a function of the plaintext, the ciphertext, and the outer subkeys $k_{1}, \ldots, k_{b-1}$ and $k_{c+1}, \ldots, k_{r}$, if we were to test for a bias as part of an exhaustive search over these outer key bits, then we would expect a bias to appear for the correct guess. In this way we can recover more key information and derive a more practical attack.

Clearly the basic building block to all these attacks will be the linear approximation, and to build a linear approximation we approximate individual components of the cipher and join these together. We will therefore use the following notation for the linear approximation of a component $f$, say, where we

write $\alpha \stackrel{f}{\rightarrow} \beta$ if $\alpha \cdot X=\beta \cdot f(X)$ with some associated bias $\epsilon$. Approximations to larger components of a block cipher, such as a round, can be written in the same way.

\subsection{Linear Cryptanalysis and SMS4}

To find a linear approximation of SMS4, we first compute the biases of all linear approximations $\alpha \stackrel{S}{\rightarrow} \beta$ to the S-box. Then we consider the evolution of a linear mask through the function $L$. For this, we define the function

$$
L_{2}(x)=x \oplus(x \ggg 2) \oplus(x \ggg 10) \oplus(x \ggg 18) \oplus(x \ggg 24)
$$

and we observe the following. Since for bit-wise rotations $\alpha \cdot(x \lll i)=(\alpha \ggg i) \cdot x$, we have for all 32-bit inputs $x$, and all linear masks $\alpha$, that $\alpha \cdot L(x)=L_{2}(\alpha) \cdot x$. 


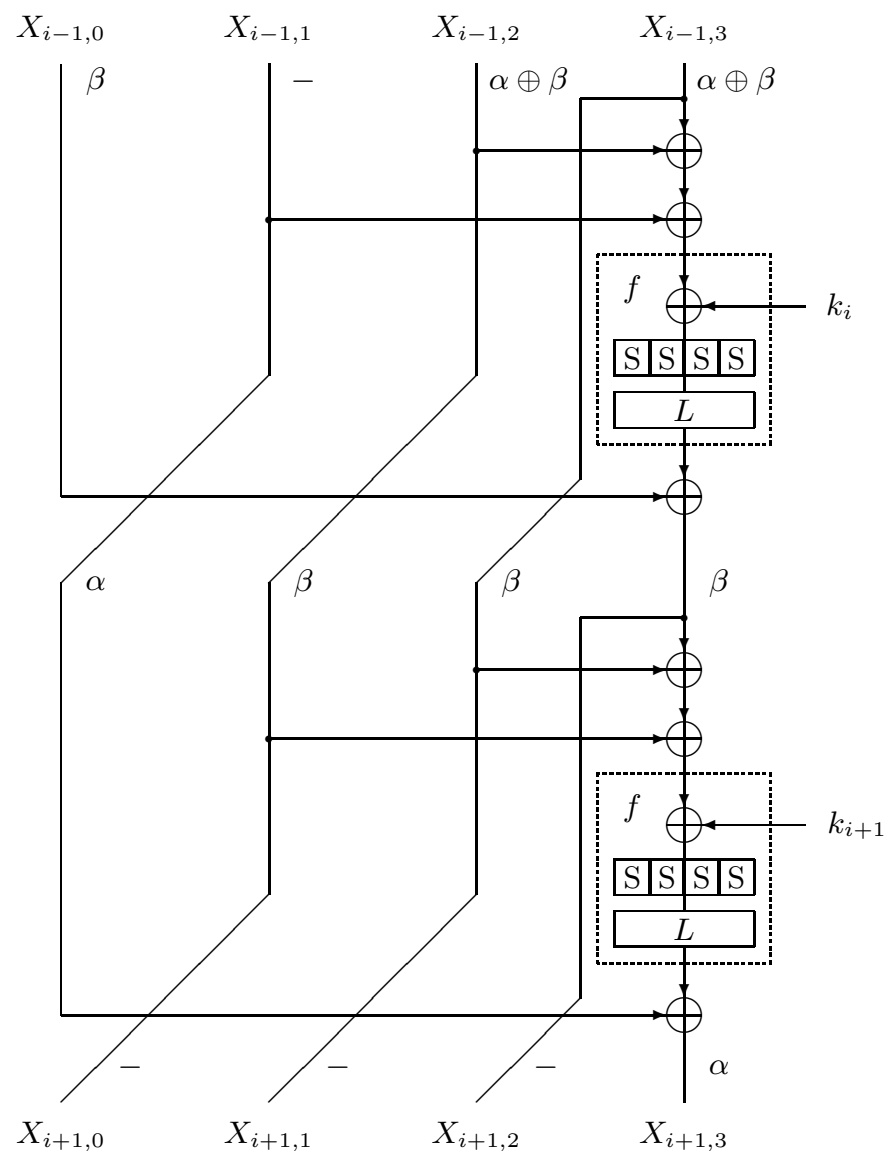

Fig. 1. Two rounds of SMS4 along with a two-round linear approximation using masks $\alpha$ and $\beta$. The input to round $i$ is $X_{i-1,0}\left\|X_{i-1,1}\right\| X_{i-1,2} \| X_{i-1,3}$.

As can be seen from Figure 1. we can identify the potential for two-round linear characteristics of the following form:

$$
(\beta, 0, \alpha \oplus \beta, \alpha \oplus \beta) \rightarrow(\alpha, \beta, \beta, \beta) \rightarrow(0,0,0, \alpha) .
$$

Such a linear approximation would require the approximation $\alpha \stackrel{f}{\rightarrow} \beta$ in the first round and $\beta \stackrel{f}{\rightarrow} \alpha$ in the second. Interestingly, by setting $\beta=\alpha$ this reduces to

$$
(\alpha, 0,0,0) \rightarrow(\alpha, \alpha, \alpha, \alpha) \rightarrow(0,0,0, \alpha)
$$

and by exploiting the structure of SMS4 in the preceeding three rounds, we derive a five-round iterative linear approximation, of which only the last two rounds are active

$$
(0,0,0, \alpha) \rightarrow(0,0, \alpha, 0) \rightarrow(0, \alpha, 0,0) \rightarrow(\alpha, 0,0,0) \rightarrow(\alpha, \alpha, \alpha, \alpha) \rightarrow(0,0,0, \alpha) .
$$


Table 1. The relevant bitmasks for the iterative linear approximations in this paper

\begin{tabular}{|cc||cc|}
\hline$\alpha$ & $L_{2}(\alpha)$ & $\alpha$ & $L_{2}(\alpha)$ \\
\hline 0x0011ffba & 0x0084be2f & 0x007852b3 & 0x00582b15 \\
0x007905e1 & 0x005afbc6 & 0x00a1b433 & 0x00f1027a \\
0x00edca7c & 0x0083ffaa & 0x00fa7099 & 0x00d20b1d \\
0x05e10079 & 0xfbc6005a & 0x11ffba00 & 0x84be2f00 \\
0x3300a1b4 & 0x7a00f102 & 0x52b30078 & 0x2b150058 \\
0x709900fa & 0x0b1d00d2 & 0x7852b300 & 0x582b1500 \\
0x7905e100 & 0x5afbc600 & 0x7c00edca & 0xaa0083ff \\
0x9900fa70 & 0x1d00d20b & 0xa1b43300 & 0xf1027a00 \\
0xb3007852 & 0x1500582b & 0xb43300a1 & 0x027a00f1 \\
0xba0011ff & 0x2f0084be & 0xca7c00ed & 0xffaa0083 \\
0xe1007905 & 0xc6005afb & 0xedca7c00 & 0x83ffaa00 \\
0xfa709900 & 0xd20b1d00 & 0xffba0011 & 0xbe2f0084 \\
\hline
\end{tabular}

To identify a bit-mask $\alpha$ that yields an approximation $\alpha \stackrel{f}{\rightarrow} \alpha$ with a good bias, we use the distribution table for linear approximations of the S-box. In this way we can list 24 different $\left(\alpha, L_{2}(\alpha)\right)$ pairs, where $L_{2}(\alpha)$ gives the mask for the output from the S-boxes, and each of these 24 five-round linear approximations holds with a bias of $\frac{7}{32768} \approx 2^{-10.2}$. These are given in Table 1

\subsection{A Distinguisher for 18-Round SMS4}

It is straightforward to see that a classical application of linear cryptanalysis gives us an 18-round distinguisher for SMS4. We can concatenate three of the five-round iterative approximations to give the following 18-round linear approximation with bias $\epsilon_{1}$ :

$$
\begin{aligned}
(0,0,0, \alpha) \stackrel{5 \text { rounds }}{\longrightarrow}(0,0,0, \alpha) \stackrel{5 \text { rounds }}{\longrightarrow}(0,0,0, \alpha) \\
\stackrel{5 \text { rounds }}{\longrightarrow}(0,0,0, \alpha) \rightarrow(0,0, \alpha, 0) \rightarrow(0, \alpha, 0,0) \rightarrow(\alpha, 0,0,0)
\end{aligned}
$$

To combine linear approximations, and to estimate the resultant bias, it is typical to appeal to the so-called piling-up lemma [12. The suitability of applying the piling-up lemma depends on the algorithms in question; for some, such as DES [15, it gives accurate results while for others, such as RC5 [18], an interround dependence means that the piling-up lemma can be misleading [19. This problem can be particularly acute when we have two consecutive active rounds. However, experimental results below suggest that the piling-up lemma should remain a reasonable tool to use with SMS4. We therefore estimate the resultant bias of the 18-round linear approximation to be $\epsilon_{1}=\left(2^{-10.2}\right)^{6} \times 2^{5}=2^{-56.2}$. This means that if we were to use $\epsilon_{1}^{-2}=2^{112.4}$ known plaintexts then we would expect our distinguisher to identify non-ideal behaviour in the reduced-round SMS4 and/or to recover a single bit of key information with a success rate of $97.7 \%$ [12. With regards to the work effort, we need to evaluate a single bit 
and increment a single counter $2^{112.4}$ times. This will be a fraction of the work required to exhaustively search a 128-bit key.

In what follows we will use the 18-round linear approximations of the form described above, of which there are 24 (see Table1). It will therefore be convenient to refer to a generic approximation from this class as $\mathcal{A}_{\alpha}^{18}$.

Experimental Confirmation. To confirm the applicability of the piling-up lemma with the basic linear approximations that we will use, we consider the equivalent linear approximations in RED-SMS4. The bias of the best approximation over a single active round - for which the input and output mask is the same -is $2^{-5}$. So over five rounds, of which two are active, the linear approximation $(0,0,0, \alpha) \rightarrow(0,0,0, \alpha)$ with $\alpha=0 \times 040 \mathrm{c}$ would have a theoretical bias of $2^{-9}$. We extend this to give a six-round approximation

$$
(\alpha, \beta, \beta, \beta) \stackrel{1 \text { round }}{\longrightarrow}(0,0,0, \alpha) \stackrel{5 \text { rounds }}{\longrightarrow}(0,0,0, \alpha)
$$

with $\beta=0 \mathrm{x} 0406$ and a bias of $2^{-2.7}$ for $\beta \stackrel{f_{r}}{\longrightarrow} \alpha$. The resultant six-round approximation has a theoretical bias of $2^{-10.7}$ and in experiments with 100 keys using the reduced key schedule and $2^{23}$ known plaintexts, the measured bias ranged between $2^{-10.0}$ and $2^{-12.1}$ with an average of $2^{-10.7}$.

On Extending to 19 Rounds. Taking $\mathcal{A}_{\alpha}^{18}$ we can prepend a single-round linear approximation of the form $(\alpha, \beta, \beta, \beta) \rightarrow(0,0,0, \alpha)$. Here we can choose $\beta$ so as to maximise the bias of this extra round. For each of the valid $L_{2}(\alpha)$ that we identified in Section 3.1, we find that there are 125 possible values to $\beta$ that give a maximum bias of $2^{-10}$ over a single round of S-box transformations. This means that there are $125 \times 24=3000$ 19-round linear approximations with a bias of $\epsilon_{2}=\left(2^{-10.2}\right)^{6} \times 2^{-10} \times 2^{6}=2^{-65.2}$. While the bias means that such approximations aren't immediately useful to us, the large number of such approximations makes them a tempting object for more advanced analysis, see Section 5.1 .

\section{Advanced Techniques}

We now use the 18-round approximations $\mathcal{A}_{\alpha}^{18}$ to recover the full 128-bit key. Standard techniques immediately compromise 20-round SMS4, while a novel extension of the work of Collard et al 4 ] extends this to 22 rounds. In the literature notation, this constitutes a $4 \mathrm{R}$-attack for which there are few precedents.

\subsection{An Attack on 20-Round SMS4}

The classical approach to using an 18-round distinguisher is to recover key information from the two outer rounds of the cipher. We will use the linear approximations $\mathcal{A}_{\alpha}^{18}$ that have only three active S-boxes in an active round and we will need the following definition: Given mask $\alpha$, denote the restriction of 


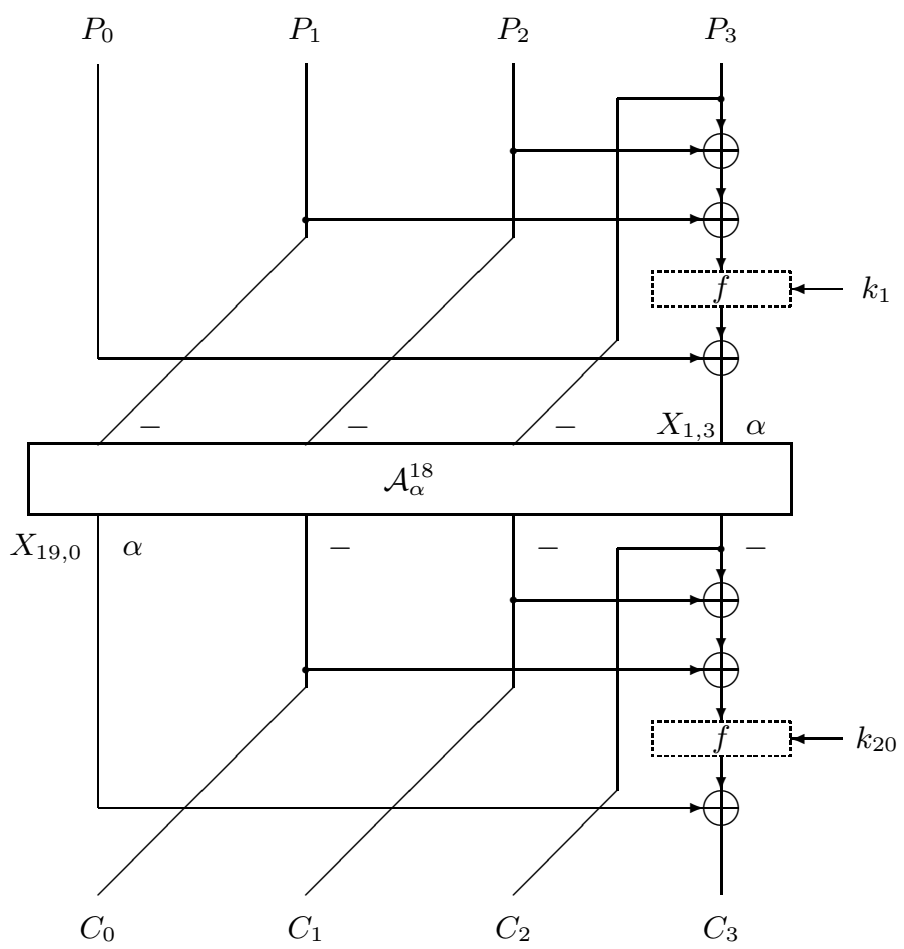

Fig. 2. Intermediate values for the $2 \mathrm{R}$ attack on 20-round SMS4

a 32-bit word y by $\alpha$ to be $R_{\alpha}(\mathrm{y})$ where $R_{\alpha}(\mathrm{y})$ consists of the deletion of bits corresponding to the inactive byte. For example, given $\alpha=0 \mathrm{x} 0011 \mathrm{ffba}$ then $R_{\alpha}(\mathrm{y})=\mathrm{y} \& 0 \mathrm{x} 00 \mathrm{ffffff}$. Note that this can be viewed as a 24-bit quantity even when the inactive byte is not in the most significant position.

It is easy to verify the following (see Figure 2). For plaintext $P_{0}\left\|P_{1}\right\| P_{2} \| P_{3}$, the bit value $\alpha \cdot X_{1,3}$ depends solely on $\alpha \cdot P_{0}, R_{\alpha}\left(P_{1} \oplus P_{2} \oplus P_{3}\right)$, and $R_{\alpha}\left(k_{1}\right)$. We can make a similar observation on the ciphertext, namely that the bit value $\alpha \cdot X_{19,0}$ depends solely on $\alpha \cdot C_{3}, R_{\alpha}\left(C_{0} \oplus C_{1} \oplus C_{2}\right)$, and $R_{\alpha}\left(k_{20}\right)$. In our 2Rattack we will recover the values of $R_{\alpha}\left(k_{1}\right)$ and $R_{\alpha}\left(k_{20}\right)$ giving 48 bits of key information. The rest of the key can be deduced using exhaustive search.

The data-related information that we need to evaluate the approximation is $\alpha \cdot P_{0}, R_{\alpha}\left(P_{1} \oplus P_{2} \oplus P_{3}\right), \alpha \cdot C_{3}$, and $R_{\alpha}\left(C_{0} \oplus C_{1} \oplus C_{2}\right)$ and we can consider a plaintext-ciphertext as being in one of $2^{50}$ possible classes according to the values of these quantities. Note that under the same key guess $R_{\alpha}\left(k_{1}\right) \| R_{\alpha}\left(k_{20}\right)$, two plaintext/ciphertext pairs from the same class yield the same values to $\alpha \cdot X_{1,3}$ and $\alpha \cdot X_{19,0}$. In [4] an efficient 1R-attack is described. We extend this approach to give a $2 \mathrm{R}$-attack recovering information from both outer rounds and adopting an optimisation that means we need only store $2^{48}$ rather than $2^{50}$ counters. 
1. Take $N=32 \epsilon^{-2}=2^{117.4}$ plaintext/ciphertext pairs.

2. Initialise a set of counters $A[0] \ldots A\left[2^{48}-1\right]$ to zero.

3. For each plaintext/ciphertext pair, compute $b=\alpha \cdot P_{0} \oplus \alpha \cdot C_{3}$ and increment $A\left[R_{\alpha}\left(P_{1} \oplus P_{2} \oplus P_{3}\right) \| R_{\alpha}\left(C_{0} \oplus C_{1} \oplus C_{2}\right)\right]$ if $b=0$ or decrement it if $b=1$, i.e.

$$
A\left[R_{\alpha}\left(P_{1} \oplus P_{2} \oplus P_{3}\right) \| R_{\alpha}\left(C_{0} \oplus C_{1} \oplus C_{2}\right)\right]+=(-1)^{\left(\alpha \cdot P_{0} \oplus \alpha \cdot C_{3}\right)} .
$$

4. For each key guess $k^{\prime}=R_{\alpha}\left(k_{1}\right) \| R_{\alpha}\left(k_{20}\right)$ keep a counter, and compute the bias generated during the attack as follows:

(a) Taking each $x=R_{\alpha}\left(P_{1} \oplus P_{2} \oplus P_{3}\right) \| R_{\alpha}\left(C_{0} \oplus C_{1} \oplus C_{2}\right)$ in turn, where $0 \leq x \leq 2^{48}-1$, compute the value

$$
c=(-1)^{\left(\alpha \cdot f ( R _ { \alpha } ( k _ { 1 } \oplus P _ { 1 } \oplus P _ { 2 } \oplus P _ { 3 } ) ) \oplus \left(\alpha \cdot f\left(R_{\alpha}\left(k_{20} \oplus C_{0} \oplus C_{1} \oplus C_{2}\right)\right)\right.\right.} .
$$

(b) Add $c \times A\left[R_{\alpha}\left(P_{1} \oplus P_{2} \oplus P_{3}\right) \| R_{\alpha}\left(C_{0} \oplus C_{1} \oplus C_{2}\right)\right]$ to the score for key guess $k^{\prime}$.

5. After recovering the 48-bit $k^{\prime}$, perform exhaustive search on the remaining 80 bits of key.

We expect to recover the right value to the 48 bits of the key by identifying the guess which gives the highest score of absolute value; using 20] the correct key should be recovered with a probability of $99.9 \%$

While the work effort for each plaintext/ciphertext pair in step 3 is much less than a round of SMS4, we might estimate the work effort for the first three steps to be equivalent to $2^{117.4} \times \frac{1}{20} \approx 2^{113.1} 20$-round SMS4 computations. The work effort for finding the right 48 bits of key material in step 4 is $2^{48} \times 2^{48}=2^{96}$ basic operations and the work to recover the rest of the key. is $2^{128-48}=2^{80}$ reduced-round SMS encryptions. One point of detail: it is possible (see below) that several keys are identified along with the correct one. However this is not uncommon, and merely extends the search for the remainder of the key.

An Optimisation. Even though the work effort for Step 4 is lower than that for data processing, we can adapt techniques introduced in 4 . Consider initialising a $\left(2^{48} \times 2^{48}\right)$ matrix $M$, where rows are indexed by $R_{\alpha}\left(k_{1}\right) \| R_{\alpha}\left(k_{20}\right)$ and the columns indexed by $R_{\alpha}\left(P_{1} \oplus P_{2} \oplus P_{3}\right) \| R_{\alpha}\left(C_{0} \oplus C_{1} \oplus C_{2}\right)$. Then the bias for the $i^{\text {th }}$ guess of $R_{\alpha}\left(k_{1}\right) \| R_{\alpha}\left(K_{20}\right)$ is given by $\sum_{j=0}^{2^{48}-1} M_{(i, j)} x_{j}$ and we can view the counters $A[\cdot]$ as a column vector $\mathbf{x}=A^{T}$. Following [4, since entry $M_{(i, j)}$ is a function of $i \oplus j$ the entire matrix $M_{(i, j)}$ can be reconstructed from a single row or column, and it is possible to compute the product $M \mathbf{x}=\mathbf{e}$ with only three products between a Discrete Fourier Transform matrix and a vector 44.This means that the complexity of generating a set of final scores for each key, represented by a vector $\mathbf{e}$ is reduced from $O\left(\left(2^{48}\right)^{2}\right)$ to $3 \times O\left(2^{48} \log _{2}\left(2^{48}\right)\right)$ [4]. The work effort for data analysis can therefore be estimated as $2^{48} \times 3 \times 48 \approx 2^{55.2}$ basic operations. 


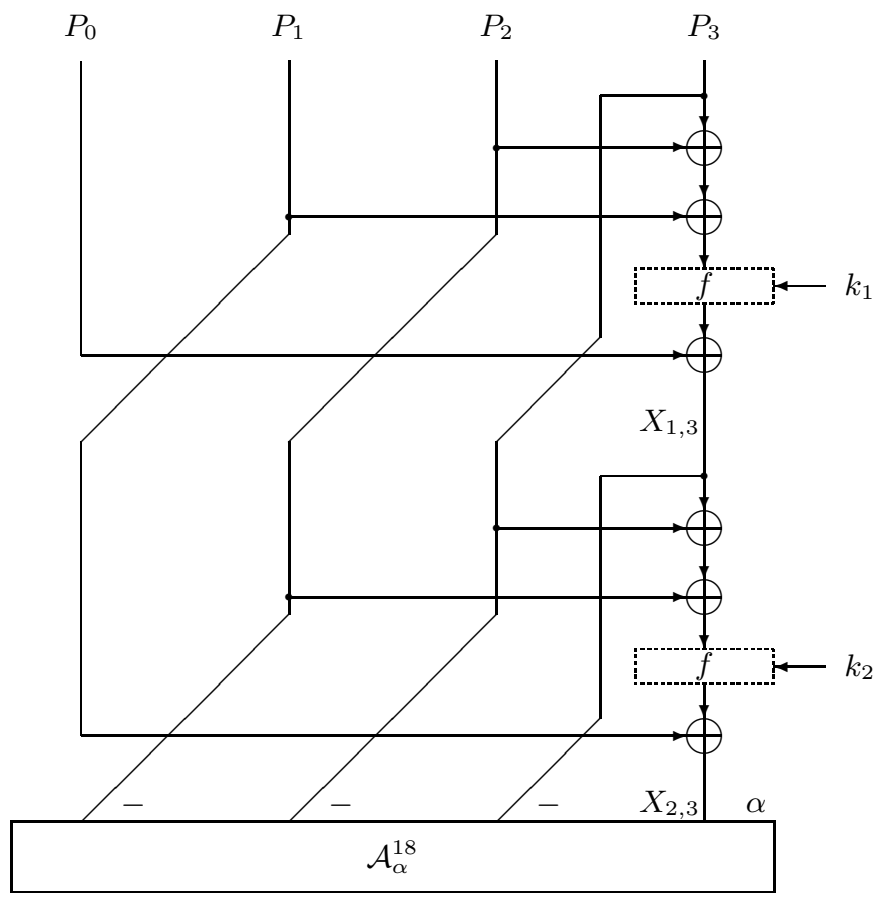

Fig. 3. The upper half of the $4 \mathrm{R}$-attack on 22-round SMS4

Experimental Confirmation. To illustrate this more advanced linear attack, we use a 10-round version of RED-SMS4 with the same linear approximation as was used in the experiments of Section 3.2 namely $(\alpha, \beta, \beta, \beta) \rightarrow(\alpha, 0,0,0)$ over nine rounds. We will recover information about $k_{10}$, though for RED-SMS4 the mask $\alpha$ we use has two inactive bytes. Recalling the bias of the approximation is $2^{-10.7}$, we take $8 \times\left(2^{11}\right)^{2}=2^{25}$ plaintexts. The data is separated according to the restriction $R_{\alpha}\left(C_{0} \oplus C_{1} \oplus C_{2}\right)$ and we perform key recovery as outlined in Section 4.1, though adapted to the $1 \mathrm{R}$-scenario. For $S_{r}$ there is a slight complication with the bit mask $\alpha$ since there are equivalent keys for one of the active nibbles. Experiments and analysis show that the best score applies to four equivalent key values, and so we recover at most six bits of $k_{10}$. With the plaintext amount we use, we theoretically have a probability of $99.9 \%$ to recover the right six bits of key [20]. In 100 experiments the correct set of keys was recovered 99 times.

\subsection{An Attack on 22-Round SMS4}

We use $\mathcal{A}_{\alpha}^{18}$ to make a 4R-attack on 22-round SMS4 (see Figure 3 for the plaintext side of the attack) and we aim to recover $k_{1}, k_{22}, R_{\alpha}\left(k_{2}\right)$ and $R_{\alpha}\left(k_{21}\right)$. For the data analysis, we will appeal to the optimisation of Collard et al. [4] described in Section 4.1 
1. We take $N=64 \epsilon^{-2}=2^{118.4}$ plaintext/ciphertext pairs.

2. View counters $A[0] \ldots A\left[2^{112}-1\right]$ as a column vector $\mathbf{x}$ and set to zero.

3. For each plaintext/ciphertext pair, compute $b=\alpha \cdot P_{1} \oplus \alpha \cdot C_{2}$ and increment $A\left[P_{1} \oplus P_{2} \oplus P_{3}\left\|R_{\alpha}\left(P_{0} \oplus P_{2} \oplus P_{3}\right)\right\| R_{\alpha}\left(C_{0} \oplus C_{1} \oplus C_{3}\right) \| C_{0} \oplus C_{1} \oplus C_{2}\right]$ if $b=0$ or decrement it if $b=1$.

4. Define (conceptually) the $\left(2^{112} \times 2^{112}\right)$ matrix $M_{(i, j)}$ where rows are indexed by the key guess $k^{\prime}=k_{1}\left\|R_{\alpha}\left(k_{2}\right)\right\| R_{\alpha}\left(k_{21}\right) \| k_{22}$ and columns indexed by $x=P_{1} \oplus P_{2} \oplus P_{3}\left\|R_{\alpha}\left(P_{0} \oplus P_{2} \oplus P_{3}\right)\right\| R_{\alpha}\left(C_{0} \oplus C_{1} \oplus C_{3}\right) \| C_{0} \oplus C_{1} \oplus C_{2}$. Recall we need only store the first column of this matrix $M_{(i, j)}$ since all values for subsequent computations can be reconstructed from a single row/column.

(a) Compute the values in the first column as $(-1)^{b}$ where

$$
\begin{array}{r}
b=\alpha \cdot f\left(R_{\alpha}\left(k_{21} \oplus C_{0} \oplus C_{1} \oplus C_{3}\right) \oplus f\left(k_{22} \oplus C_{0} \oplus C_{1} \oplus C_{2}\right)\right) \\
\oplus \alpha \cdot f\left(R_{\alpha}\left(k_{2} \oplus P_{0} \oplus P_{2} \oplus P_{3}\right) \oplus f\left(k_{1} \oplus P_{1} \oplus P_{2} \oplus P_{3}\right)\right)
\end{array}
$$

(b) Efficiently compute $M \mathbf{x}=\mathbf{e}$ using 4. This gives the right result since

$$
\begin{aligned}
\alpha \cdot X_{2,3}= & \alpha \cdot f\left(R_{\alpha}\left(k_{2}\right) \oplus R_{\alpha}\left(P_{2} \oplus P_{3} \oplus X_{1,3}\right)\right) \oplus \alpha \cdot P_{1} \\
= & \alpha \cdot f\left(R_{\alpha}\left(k_{2}\right) \oplus R_{\alpha}\left(P_{0} \oplus P_{2} \oplus P_{3}\right)\right. \\
& \left.\oplus f\left(k_{1} \oplus P_{1} \oplus P_{2} \oplus P_{3}\right)\right) \oplus \alpha \cdot P_{1}
\end{aligned}
$$

and we have a similar expression for the ciphertext side.

5. Recover the 112-bit $k^{\prime}$ from e and search the remaining bits of the key.

The only hypothesis needed to apply 4 to the 22-round attack is that the $\left(2^{112} \times 2^{112}\right)$ matrix $M_{(i, j)}$ (see optimisation to Section 4.1) should only depend on $i \oplus j$, which is the case for the expression in Step 2. We expect to recover the right value to the 112 bits of the key from the guess with the highest score of absolute value. With $2^{118.4}$ plaintexts, the method of [20] suggests that we are

\begin{tabular}{|c|c|c|c|c|c|c|c|}
\hline$r$ & texts & mem. & $\begin{array}{c}\text { work } \\
\text { steps 1-3 }\end{array}$ & $\begin{array}{c}\text { work step } 4 \\
(\mathrm{w} / \mathrm{o}[4])\end{array}$ & $\begin{array}{c}\text { work step } 4 \\
\text { (w. [4]) }\end{array}$ & $\begin{array}{c}\text { work } \\
\text { step } 5\end{array}$ & $\begin{array}{c}\text { success } \\
(\%)\end{array}$ \\
\hline 19 & $2^{116.4}$ & $2^{24}$ & $2^{112.2}$ & $2^{43.8}$ & $2^{26}$ & $2^{104}$ & 99.5 \\
\hline 20 & $2^{117.4}$ & $2^{48}$ & $2^{113.1}$ & $2^{91.7}$ & $2^{50.9}$ & $2^{80}$ & 99.9 \\
\hline 21 & $2^{117.4}$ & $2^{80}$ & $2^{113.0}$ & $\left(2^{155.6}\right)$ & $2^{83.5}$ & $2^{48}$ & 84.8 \\
\hline 21 & $2^{118.4}$ & $2^{80}$ & $2^{114.0}$ & $\left(2^{155.6}\right)$ & $2^{83.5}$ & $2^{48}$ & 99.9 \\
\hline 22 & $2^{117.4}$ & $2^{112}$ & $2^{112.9}$ & $\left(2^{219.5}\right)$ & $2^{115.9}$ & $2^{16}$ & 17.7 \\
\hline 22 & $2^{118.4}$ & $2^{112}$ & $2^{113.9}$ & $\left(2^{219.5}\right)$ & $2^{115.9}$ & $2^{16}$ & 99.9 \\
\hline
\end{tabular}
very likely to recover the correct value, see Table 2. The work effort for Steps

Table 2. The estimated work efforts for a range of linear cryptanalytic attacks on $r$-round SMS4 for $19 \leq r \leq 22$. Work is estimated in terms of the number of $r$-round encryptions (for appropriate $r$ ) with that exceeding $2^{128}$ placed in parentheses. 
1 -3 can be estimated as $2^{118.4} \times \frac{1}{22} \approx 2^{113.9} 22$-round SMS4 computations while the effort in Step 4 is approximately $2^{112} \times 3 \times 112 \times \frac{1}{22} \approx 2^{115.9} 22$-round SMS4 computations, and this dominates the attack.

\section{Ongoing and Future Research}

It is natural to consider some more advanced techniques in trying to attack more rounds of SMS4. In this section we consider the use of multiple linear approximations as well as the use of chosen-plaintexts.

\subsection{Multiple Linear Approximations}

Multiple linear approximations were first proposed in [67] and they have been the subject of much recent analysis 35 . Here we take $m$ different linear approximations, where we use $\kappa_{j}$ to denote a single bit of key information,

$$
\alpha_{j} \cdot P \oplus \beta_{j} \cdot C=\kappa_{j} .
$$

The purpose is to use several approximations to reduce the number of plaintexts when keeping the same probability of sucess. Let $\epsilon^{j}$ denote the theoretical bias of the $j^{\text {th }}$ approximation and let $e_{k_{\text {outer }}}^{j}$ denote the experimental bias of the $j^{\text {th }}$ approximation observed when using the guess $k_{\text {outer }}$ for the outer key bit:2 . If, with sufficiently many plaintexts, we compute

$$
\min _{k_{\text {outer }}} \min _{\left(\kappa_{1}, \ldots, \kappa_{m}\right) \in\{0,1\}^{m}} \sum_{j=1}^{m}\left(\epsilon^{j}-(-1)^{\kappa_{j}} e_{k_{\text {outer }}^{j}}\right)^{2},
$$

then the minimum value will be given by the correct values of $k_{\text {outer }}$ and the correct values of the $m$ bits of internal key represented by $\left(\kappa_{1}, \ldots, \kappa_{m}\right)$.

A straightforward application of this method needs $2^{|k|+m}$ computations. However this can be reduced if we introduce $\sigma_{k}^{j}$ where $\sigma_{k}^{j}=1$ if $\operatorname{sgn}\left(\epsilon^{j}\right)=\operatorname{sgn}\left(e_{k}^{j}\right)$ and zero otherwise. Then we observe that, for each $j$,

$$
\min _{\kappa_{j} \in\{0,1\}}\left(\epsilon^{j}-(-1)^{\kappa_{j}} e_{k}^{j}\right)^{2}=\left(\epsilon^{j}-(-1)^{\sigma_{k}^{j}} e_{k}^{j}\right)^{2}
$$

and so we have the equality

$$
\begin{aligned}
& \min _{k_{\text {outer }}} \min _{\left(\kappa_{1}, \ldots, \kappa_{m}\right) \in(0,1)^{m}} \sum_{j=1}^{m}\left(\epsilon^{j}-(-1)^{\kappa_{j}} e_{k_{\text {outer }}^{j}}^{j}\right)^{2} \\
& =\min _{k_{\text {outer }}} \sum_{j=1}^{m}\left(\epsilon^{j}-(-1)^{\sigma_{k_{\text {outer }}}} e_{k_{\text {outer }}}^{j}\right)^{2} .
\end{aligned}
$$

This requires $m 2^{\left|k_{\text {outer }}\right|}$ computations, though we only recover the correct value to $k_{\text {outer }}$. However this is usually the most important block of key information to recover.

\footnotetext{
${ }^{2}$ An equivalent approach considers the imbalance which is double the bias 3 .
} 
Application to SMS4. To gauge the possible limits of linear cryptanalysis, we will optimistically assume that the gain that can be made when using multiple linear approximations is linear in the number of approximations. We will then use the techniques above to combine a set of different linear 19-round approximations and illustrate the basis for a possible attack on 23-round SMS4.

To do this we need a set of linear approximations and we will choose 125 19-round approximations $\mathcal{A}_{\alpha \beta}^{19}$ where these are the extensions of a given, fixed, 18 -round distinguisher $\mathcal{A}_{\alpha}^{18}$ by the 125 choices for $\beta$. (These approximations were identified in Section 3.2). We denote by $\epsilon$ the theoretical bias of $2^{-65.2}$ which is the same for each of the $\mathcal{A}_{\alpha \beta}^{19}$.

1. Take $N=2^{125.4}$ plaintext/ciphertext pairs.

2. For each $\beta$ view counters $A[\beta][0], \ldots, A[\beta]\left[2^{112}-1\right]$ as a column vector $\mathbf{x}^{\beta}$ and set this to zero.

3. For each $\beta$ and each plaintext/ciphertext pair, compute $b=\alpha \cdot P_{2} \oplus \beta \cdot P_{0} \oplus$ $\beta \cdot P_{1} \oplus \beta \cdot P_{3} \oplus \alpha \cdot C_{2}$ and increment $A[\beta]\left[P_{1} \oplus P_{2} \oplus P_{3} \| R_{\alpha}\left(P_{0} \oplus P_{2} \oplus\right.\right.$ $\left.\left.P_{3}\right)\left\|R_{\alpha}\left(C_{0} \oplus C_{1} \oplus C_{3}\right)\right\| C_{0} \oplus C_{1} \oplus C_{2}\right]$ if $b=0$ or decrement it if $b=1$.

4. Define for each $\beta$ (conceptually) the $\left(2^{112} \times 2^{112}\right)$ matrix $M_{(i, j)}^{\beta}$ where rows are indexed by the outer key guess $k_{\text {outer }}=k_{1}\left\|R_{\alpha}\left(k_{2}\right)\right\| R_{\alpha}\left(k_{21}\right) \| k_{22}$ and columns indexed by $x=P_{1} \oplus P_{2} \oplus P_{3}\left\|R_{\alpha}\left(P_{0} \oplus P_{2} \oplus P_{3}\right)\right\| R_{\alpha}\left(C_{0} \oplus C_{1} \oplus\right.$ $\left.C_{3}\right) \| C_{0} \oplus C_{1} \oplus C_{2}$. Recall we need only store the first column of this matrix $M_{(i, j)}^{\beta}$ since all values for subsequent computations can be reconstructed from a single row/column.

5. Compute the values in the first column as $(-1)^{b}$ where

$$
\begin{aligned}
b= & \alpha \cdot f\left(R_{\alpha}\left(k_{21} \oplus C_{0} \oplus C_{1} \oplus C_{3}\right) \oplus f\left(k_{22} \oplus C_{0} \oplus C_{1} \oplus C_{2}\right)\right) \\
& \oplus \beta \cdot f\left(R_{\alpha}\left(k_{2} \oplus P_{0} \oplus P_{2} \oplus P_{3}\right) \oplus f\left(k_{1} \oplus P_{1} \oplus P_{2} \oplus P_{3}\right)\right)
\end{aligned}
$$

6. Efficiently compute $M^{\beta} \mathbf{x}^{\beta}=\mathbf{e}^{\beta}$ using [4].

7. For each guess to $k_{\text {outer }}$, compute $\sum_{\beta}\left(\epsilon-(-1)^{\sigma_{k_{\text {outer }}}^{\beta}} e_{k_{\text {outer }}}^{\beta}\right)^{2}$.

8. Assume that the minimum value is given by the correct guess for the 112-bit $k_{\text {outer }}$ and then search the remaining bits of the key.

The work effort for this attack is dominated by Step 3. To derive the maximum number of plaintexts we can use, we observe that the work effort of Step 3 can be expressed as $\frac{125 \times N}{23} 23$-round SMS4 computations. To give an academic attack, we need this to be less than $2^{128}$ 23-round SMS4 computations and so we have $N \leq \frac{23 \times 2^{128}}{125} \approx 2^{125.4}$ for a valid attack.

However $2^{125.4}$ corresponds to around $4 \times \frac{\left(2^{65.2}\right)^{2}}{125}$, but since we are recovering 112 bits of key information the success rate 20] will be almost negligible. Thus while it is conceivable that 23 rounds could be attacked (academically) we feel that this is somewhat optimistic.

Unfortunately the reduced version of SMS4 used earlier doesn't exhibit different linear approximations with the same bias. Intead we were able to experiment on a different reduced cipher design, but it was too far-removed from SMS4 for us 
to be able to draw any substantive conclusions. Our experiments demonstrated improvements to the number of plaintexts required in a successful attack, but the probability of success was somewhat less than anticipated by theory. We therefore leave it as an object of future research to provide a sound estimate for the effectiveness of multiple linear approximations on SMS4.

\subsection{On Using Chosen Plaintext}

Several extensions to linear cryptanalysis consider the use of chosen plaintext. One of these is described by Knudsen and Mathiassen [8. In early work for this paper we considered using variants of this technique and at first sight it seemed to be well-suited to SMS4. However technical complications meant that it was hard to use these techniques directly with the 18-round distinguisher and we were unable to get any satisfactory advantages. We also considered using differentiallinear cryptanalysis [9] but our preliminary conclusion was somewhat negative. We therefore leave it as an open problem to decide whether chosen plaintext can give any real advantage over the typical known plaintext approach.

\section{Conclusions}

In this paper we have considered the cryptanalysis of the block cipher SMS4. The cipher is both actively deployed and of an elegant and simple design, making it of considerable interest to the cryptanalyst. While much of the preceeding work is concentrated on the differential cryptanalysis of SMS4, by turning to linear cryptanalysis we have demonstrated some simple and effective attacks. These yield results which are superior to all previous claims and which, therefore, give the best current attacks on SMS4.

\section{References}

1. Biham, E., Shamir, A.: Differential Cryptanalysis of the Data Encryption Standard. Springer, Heidelberg (1993)

2. Bogdanov, A., Knudsen, L.R., Leander, G., Paar, C., Poschmann, A., Robshaw, M.J.B., Seurin, Y., Vikkelsoe, C.: PRESENT: An ultra-lightweight block cipher. In: Paillier, P., Verbauwhede, I. (eds.) CHES 2007. LNCS, vol. 4727, pp. 450-466. Springer, Heidelberg (2007)

3. Biryukov, A., De Cannière, C., Quisquater, M.: On multiple linear approximations. In: Franklin, M. (ed.) CRYPTO 2004. LNCS, vol. 3152, pp. 1-22. Springer, Heidelberg (2004)

4. Collard, B., Standaert, F.-X., Quisquater, J.-J.: Improving the time complexity of matsui's linear cryptanalysis. In: Nam, K.-H., Rhee, G. (eds.) ICISC 2007. LNCS, vol. 4817, pp. 77-88. Springer, Heidelberg (2007)

5. Collard, B., Standaert, F.-X., Quisquater, J.-J.: Experiments on the multiple linear cryptanalysis of reduced round serpent. In: Nyberg, K. (ed.) FSE 2008. LNCS, vol. 5086, pp. 382-397. Springer, Heidelberg (2008) 
6. Kaliski, B.S., Robshaw, M.J.B.: Linear cryptanalysis using multiple approximations. In: Desmedt, Y.G. (ed.) CRYPTO 1994. LNCS, vol. 839, pp. 26-39. Springer, Heidelberg (1994)

7. Kaliski, B.S., Robshaw, M.J.B.: Linear Cryptanalysis and FEAL. In: Preneel, B. (ed.) FSE 1994. LNCS, vol. 1008, pp. 249-264. Springer, Heidelberg (1995)

8. Knudsen, L., Mathiassen, J.: A chosen-plaintext linear attack on DES. In: Schneier, B. (ed.) FSE 2000. LNCS, vol. 1978, pp. 262-272. Springer, Heidelberg (2001)

9. Langford, S.K., Hellman, M.E.: Differential-linear cryptanalysis. In: Desmedt, Y.G. (ed.) CRYPTO 1994. LNCS, vol. 839, pp. 17-25. Springer, Heidelberg (1994)

10. Liu, F., Ji, W., Hu, L., Ding, J., Lv, S., Pyshkin, A., Weinmann, R.-P.: Analysis of the SMS4 block cipher. In: Pieprzyk, J., Ghodosi, H., Dawson, E. (eds.) ACISP 2007. LNCS, vol. 4586, pp. 158-170. Springer, Heidelberg (2007)

11. Lu, J.: Attacking reduced-round versions of the SMS4 block cipher in the chinese WAPI standard. In: Qing, S., Imai, H., Wang, G. (eds.) ICICS 2007. LNCS, vol. 4861, pp. 306-318. Springer, Heidelberg (2007)

12. Matsui, M.: Linear cryptanalysis method for DES cipher. In: Helleseth, T. (ed.) EUROCRYPT 1993. LNCS, vol. 765, pp. 386-397. Springer, Heidelberg (1994)

13. Matsui, M.: The first experimental cryptanalysis of the data encryption standard. In: Desmedt, Y.G. (ed.) CRYPTO 1994. LNCS, vol. 839, pp. 1-11. Springer, Heidelberg (1994)

14. Murphy, S.: The Independence of Linear Approximations in Symmetric Cryptanalysis. IEEE Transactions on Information Theory 52, 5510-5518 (2006)

15. National Institute of Standards and Technology. FIPS 46-3: Data Encryption Standard (November 1998), http://csrc.nist.gov

16. National Institute of Standards and Technology. FIPS 197: Advanced Encryption Standard (November 2001), http://csrc.nist.gov

17. Nyberg, K.: Linear approximation of block ciphers. In: De Santis, A. (ed.) EUROCRYPT 1994. LNCS, vol. 950, pp. 439-444. Springer, Heidelberg (1995)

18. Rivest, R.L.: The RC5 Encryption Algorithm. In: Preneel, B. (ed.) FSE 1994. LNCS, vol. 1008, pp. 86-96. Springer, Heidelberg (1995)

19. Selçuk, A.A.: New results in linear cryptanalysis of RC5. In: Vaudenay, S. (ed.) FSE 1998. LNCS, vol. 1372, pp. 1-16. Springer, Heidelberg (1998)

20. Selçuk, A.: On Probability of Success in Linear and Differential Cryptanalysis. Journal of Cryptology 21(1), 131-147 (2008)

21. Tardy-Corfdir, A., Gilbert, H.: A known plaintext attack of FEAL-4 and FEAL-6. In: Feigenbaum, J. (ed.) CRYPTO 1991. LNCS, vol. 576, pp. 172-182. Springer, Heidelberg (1992)

22. Zhang, L., Zhang, W., Wu, W.: Cryptanalysis of reduced-round SMS4 block cipher. In: Mu, Y., Susilo, W., Seberry, J. (eds.) ACISP 2008. LNCS, vol. 5107, pp. 216-229. Springer, Heidelberg (2008) 